\title{
Modelos de dimensão finita para a estimação de parâmetros genéticos para a produção de leite de primeiras lactações de vacas da raça Holandesa
}

\author{
Finite dimension models to estimate genetic parameters for first lactation milk yields of \\ Holstein cows
}

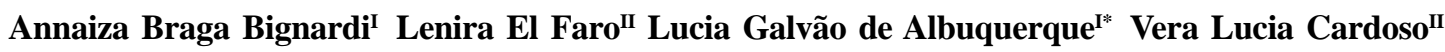 \\ Paulo Fernando MachadoIII
}

\section{RESUMO}

Foram estimados parâmetros genéticos para produção de leite acumulada até 305 dias (P305) e produção de leite no dia do controle (PLDC) de 50.171 controles mensais de 9.281 primeiras lactações de vacas da raça Holandesa. A P305 e as PLDC foram analisadas por meio de modelo animal uni e bicaracterísticas. Para a P305 o modelo incluiu como aleatório, o efeito genético e como efeitos fixos o grupo de contemporâneos e a covariável idade da vaca ao parto. Para as PLDC foi usado o mesmo modelo descrito para a P305, incluindo como covariável o número de dias em lactação. Os componentes de variância foram estimados pelo Método da Máxima Verossimilhança Restrita. As estimativas de herdabilidade $\left(h^{2}\right)$ para as PLDC oscilaram entre 0,07 e 0,19 em análises unicaracterísticas e, de 0,12 a 0,22 nas bicaracterísticas. Para a P305, as $h^{2}$ resultantes das análises uni-característica e bicaracterística foram 0,26 e 0,27, respectivamente. As correlações genéticas das PLDC com a P305 foram todas positivas e elevadas, variando de 0,63 a 1,00. As correlações genéticas entre as PLDC variaram de 0,30 a 1,00. A seleção para a $P 305$ parece ser o melhor critério de seleção a ser adotado, uma vez que proporciona maiores ganhos genéticos para as produções de leite em, praticamente, todos os controles da lactação.

Palavras-chave: critério de seleção, herdabilidade, resposta correlacionada.

\section{ABSTRACT}

Genetic parameters for 50,171 first lactation testday milk yields and 305 day milk yield (Y305) of 9,281 Holstein cows were estimated, applying uni and bi-trait animal models. The model for Y305 included the additive genetic effect as random and the fixed effects of contemporary group and age of cow at calving as covariable. For TDMY the same animal model described for Y305 was used, including days in milk as covariable. Variance components were estimated by Restricted Maximum Likelihood. Heritability estimates obtained for TDMY ranged from 0.07 to 0.19 and from 0.12 to 0.22 by uni-trait and bi-trait analysis, respectively. Heritability for Y305 was 0.26 by uni-trait and 0.27 by bi-trait analysis. The genetic correlations between TDMY and Y305 were all positive and high, ranging from 0.63 to 1.00. The genetic correlations between TDMY ranged from 0.30 to 1.00. Selection for Y305 seems to be the best selection criterion to be adopted, since it provides larger genetic gain for milk productions in, practically, all test days.

Key words: correlated response, heritability, selection criteria.

\section{INTRODUÇÃO}

A produção de leite no dia do controle (PLDC) é definida como a somatória de todas as pesagens de leite de uma vaca, durante o período de 24 horas (PANDER et al., 1992; SCHAEFFER \& JAMROZIK, 1996; FIRAT et al., 1997). A utilização da PLDC em substituição à produção acumulada até 305 dias (P305) tem sido proposta como critério de seleção. Para tanto, têm sido considerados modelos denominados "test-day models" (TDM), que consideram todos os efeitos genéticos e ambientais relacionados com as PLDC (PTAK \& SCHAEFFER, 1993; SWALVE, 1995).

Os TDM podem ser classificados como de dimensão finita ou de dimensão infinita, que são os

\footnotetext{
IDepartamento de Zootecnia, Faculdade de Ciências Agrárias e Veterinárias, FCAV/UNESP. Via de Acesso Prof. Paulo Donato Castellane, s/n, 14884-900. Jaboticabal, SP, Brasil. E-mail:lgalb@fcav.unesp.br. *Autor para correspondência.

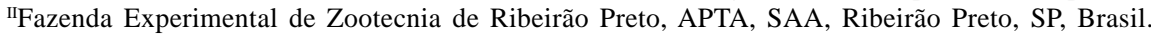

"IIDepartamento de Zootecnia, Escola Superior de Agricultura “Luiz de Queiroz” (ESALQ), Universidade de São Paulo (USP), Piracicaba, SP, Brasil.
} 
modelos de regressão aleatória (MEYER, 1998). Embora os modelos de regressão aleatória sejam indicados para análise de dados longitudinais, como a produção de leite no dia do controle, os TDM ordinários ainda estão sendo empregados para avaliação genética de reprodutores para produção de leite em vários países (INTERBULL, 2007). Em geral, o uso da PLDC para a avaliação genética de animais permite quantificar fatores específicos de cada dia de controle, tais como número de ordenhas, duração do período seco, duração do período de serviço anterior e corrente, prenhez ou doença, os quais mudam não somente de animal para animal, mas também de um controle para outro. Outra vantagem em relação à produção aos 305 dias é o fato de dispensar o uso de projeção de lactações parciais para produção total (EL FARO \& ALBUQUERQUE, 2005), visto que os fatores de projeção assumem uma curva de lactação padrão. Com os TDM é possível a inclusão de lactações incompletas, aumentando o número de filhas por touro, proporcionando, conseqüentemente, uma maior confiabilidade nos testes de touros (MEYER et al., 1989; SWALVE, 1998). Com os modelos para PLDC também é possível obter o encurtamento do intervalo de gerações (FERREIRA et al., 2003). Os resultados obtidos para os TDM de dimensão finita têm indicado maior variabilidade genética no meio da lactação e maiores herdabilidades para as PLDC que para a produção acumulada até os 305 dias (SWALVE, 1995; MELO et al., 2005).

O objetivo deste trabalho foi estimar parâmetros genéticos para a produção de leite de primeiras lactações de vacas da raça Holandesa, comparando as produções de leite no dia do controle e a produção de leite acumulada até os 305 dias como critérios de seleção.

\section{MATERIAL E MÉTODOS}

Foram analisados 50.171 controles mensais de produção de leite de 9.281 primeiras lactações de vacas da raça Holandesa, filhas de 625 touros, provenientes de 93 rebanhos dos Estados de São Paulo e Minas Gerais. Os dados foram provenientes do Programa de Análise de Rebanhos Leiteiros e da Qualidade do Leite da ESALQ - USP, no período de 1996 a 2003. As lactações foram truncadas aos 305 dias e os controles mensais de produção de leite, obtidos entre cinco e 305 dias após o parto, foram divididos em intervalos de, aproximadamente, 30 dias, totalizando dez controles (PLDC1 a PLDC10). Foram eliminadas vacas com idade ao parto inferior a 18 ou superior a 48 meses e para a P305 foram excluídas lactações com duração menor que 180 dias. O grupo de contemporâneos para a P305 foi definido como rebanho-ano-estação do parto e para as PLDC foi definido como rebanho-ano-mês do controle e, para ambas as características, aplicou-se a restrição de que cada grupo deveria conter, no mínimo, quatro observações. Um resumo da estrutura dos dados é apresentado na tabela 1.

As características foram analisadas por meio de modelo animal, em análises uni e bicaracterísticas, sendo que as análises bicaracterísticas foram realizadas entre as dez PLDC e entre estas e a P305, totalizando 66 análises. Em todas as análises foi utilizada a mesma matriz de parentesco.

Para verificar a influência dos efeitos fixos sobre as características analisadas, foram realizadas análises preliminares, pelo método de quadrados mínimos. Para as PLDC, o modelo linear incluiu os efeitos de idade da vaca ao parto (linear e quadrática) e do número de dias em lactação (linear), além do grupo de contemporâneos. Para a P305, foi incluída a duração da lactação no modelo, como covariável, além do grupo de contemporâneos e da idade ao parto, entretanto, o seu efeito não foi significativo.

Os modelos mistos para a P305 e as PLDC podem ser representados em sua forma matricial por:

$\mathrm{y}=\mathrm{Xb}+\mathrm{Za}+\mathrm{e}$, em que: $\mathrm{y}=$ é o vetor das produções de leite acumuladas até os 305 dias e em cada controle leiteiro; $b$ = é o vetor de soluções para os efeitos fixos; a = é o vetor de soluções para os efeitos aleatórios genéticos aditivos; $\mathrm{X}$ e $\mathrm{Z}=$ são as matrizes de incidência, para os efeitos fixos e aleatório de animal, respectivamente e e= é o vetor do efeito aleatório residual.

Os componentes de (co)variâncias, necessários à estimação dos parâmetros genéticos, foram estimados pelo método da máxima verossimilhança restrita, que utiliza algoritmo livre de derivadas (MTDFREML), desenvolvido por BOLDMAN et al. (1995).

O ganho genético e a resposta correlacionada para seleção foram obtidos utilizando as estimativas de herdabilidade das análises bicaracterísticas, as correlações genéticas e o desvio padrão fenotípico, considerando-se uma mesma intensidade de seleção (igual à unidade). O ganho genético, a resposta correlacionada para seleção e a eficiência relativa de seleção foram calculadas por uma fórmula usual de índice de seleção, considerando um teste de progênie com número de filhas por touro variando de cinco a 200(VAN VLECK, 1993).

\section{RESULTADOS E DISCUSSÃO}

As médias observadas, os desvios-padrão e os coeficientes de variação para as produções de 
Tabela 1 - Resumo da estrutura dos dados, número de animais (N), média, desvio-padrão (DP) e coeficientes de variação (CV\%) para as produções de leite no dia do controle (PLDC) e produção de leite acumulada até 305 dias (P305) e médias de dias em lactação (DEL).

\begin{tabular}{|c|c|c|c|c|c|c|}
\hline \multirow{2}{*}{ Informação } & \multirow{2}{*}{$\mathrm{N}$} & \multicolumn{3}{|c|}{------Produção de leite (Kg)------ } & \multicolumn{2}{|c|}{------DEL------ } \\
\hline & & Média & DP & CV (\%) & Média & $\mathrm{DP}$ \\
\hline PLDC1 & 5959 & 22,47 & 7,13 & 31,75 & 16,69 & 5,33 \\
\hline PLDC2 & 5972 & 27,49 & 7,04 & 25,63 & 44,69 & 5,68 \\
\hline PLDC3 & 5579 & 28,58 & 6,98 & 24,41 & 74,92 & 5,54 \\
\hline PLDC4 & 5503 & 28,66 & 7,13 & 24,89 & 104,96 & 5,68 \\
\hline PLDC5 & 5127 & 28,86 & 7,30 & 25,32 & 134,93 & 5,48 \\
\hline PLDC6 & 4923 & 28,20 & 7,50 & 26,48 & 164,94 & 5,48 \\
\hline PLDC7 & 4729 & 27,48 & 7,73 & 28,13 & 194,90 & 5,43 \\
\hline PLDC8 & 4376 & 26,60 & 7,77 & 29,21 & 224,87 & 5,24 \\
\hline PLDC9 & 4220 & 25,30 & 7,81 & 30,90 & 254,83 & 5,24 \\
\hline PLDC10 & 3802 & 22,96 & 8,31 & 36,19 & 284,60 & 5,91 \\
\hline P305 & 6207 & 7408,14 & 1847,72 & 24,94 & 280,30 & 29,27 \\
\hline Rebanhos & 93 & & & & & \\
\hline Touros & 625 & & & & & \\
\hline Animais em A & 17.688 & & & & & \\
\hline
\end{tabular}

$\mathrm{A}=$ matriz de parentesco.

leite no dia do controle (PLDC1 até PLDC10) e para produção de leite até 305 dias (P305) encontram-se na tabela 1. As PLDC apresentaram um aumento da produção de leite no início $(22,74 \mathrm{~kg})$, até o pico de lactação (28,86kg), ocorrido entre 75 e 135 dias de lactação, decrescendo posteriormente até o final da lactação (22,96kg), demonstrando assim a forma típica da curva de lactação. A média para a P305 foi de 7.408,14kg por lactação, próxima da média geral da raça Holandesa, que, segundo o arquivo zootécnico nacional, foi de $7.571,00 \mathrm{~kg}$ por lactação (ARQUIVO ZOOTÉCNICONACIONAL DEGADODELEITE, 2007).
As estimativas dos componentes de variância genética, residual e fenotípica e dos coeficientes de herdabilidade para PLDC e P305, para as análises unicaracterísticas, estão apresentadas na tabela 2. Como se pode observar, as variâncias genéticas aumentaram do início $\left(4,86 \mathrm{~kg}^{2}\right)$ até o terceiro mês $\left(6,25 \mathrm{~kg}^{2}\right)$, diminuíram do quarto ao sexto mês e, aumentaram gradativamente a partir do sétimo mês $\left(5,60 \mathrm{~kg}^{2}\right)$ até o final da lactação $\left(8,20 \mathrm{~kg}^{2}\right)$. As variâncias fenotípicas foram mais elevadas no início da lactação, decresceram até o quinto mês de lactação, aumentando a partir do sexto mês até o final da lactação. Para as

Tabela 2 - Estimativas dos componentes de variância genética aditiva $\left(\sigma_{\mathrm{a}}^{2}\right)$, residual $\left(\sigma_{\mathrm{e}}^{2}\right)$, fenotípica $\left(\sigma_{\mathrm{p}}^{2}\right)$ e herdabilidades $\left(\mathrm{h}^{2}\right)$, para as produções de leite no dia do controle (PLDC) e para produção de leite acumulada até 305 dias (P305), em análises unicaracterísticas.

\begin{tabular}{lcccr}
\hline Características & $\sigma^{2}{ }_{\mathrm{a}}$ & $\sigma_{\mathrm{e}}^{2}$ & $\sigma_{\mathrm{p}}^{2}$ & $\mathrm{~h}^{2}$ \\
\hline PLDC1 & 4,86 & 37,79 & 42,66 & $0,11 \pm 0,027$ \\
PLDC2 & 5,52 & 30,72 & 36,24 & $0,15 \pm 0,031$ \\
PLDC3 & 6,25 & 26,35 & 32,60 & $0,19 \pm 0,034$ \\
PLDC4 & 4,43 & 27,15 & 31,58 & $0,14 \pm 0,031$ \\
PLDC5 & 4,67 & 27,18 & 31,85 & $0,15 \pm 0,034$ \\
PLDC6 & 2,46 & 30,78 & 33,25 & $0,07 \pm 0,029$ \\
PLDC7 & 5,60 & 30,29 & 35,89 & $0,16 \pm 0,036$ \\
PLDC8 & 6,11 & 29,86 & 35,97 & $0,17 \pm 0,038$ \\
PLDC9 & 5,96 & 32,10 & 38,05 & $0,16 \pm 0,037$ \\
PLDC10 & 8,20 & 41,25 & 49,45 & $0,17 \pm 0,039$ \\
P305 & 439474,88 & 1263984,35 & 1703459,23 & $0,26 \pm 0,000$ \\
\hline
\end{tabular}

Ciência Rural, v.38, n.6, set, 2008. 
PLDC1 e PLDC10, estas foram mais elevadas que para os demais controles, mesma tendência observada para coeficientes de variação. A mesma tendência das variâncias genéticas foi observada para as variâncias residuais. A magnitude das estimativas das variâncias residuais, para todos os controles mensais de produção, sugere a existência de grandes diferenças de meio ambiente entre os sistemas de produção no presente estudo.

As estimativas das herdabilidades $\left(\mathrm{h}^{2}\right)$ para as PLDC, provenientes das análises unicaracterísticas, oscilaram entre 0,07 e 0,19 (Tabela 2), sendo que o maior valor foi obtido para o terceiro controle $(0,19)$. As $h^{2}$ foram de magnitude menor que as relatadas na literatura, pois FIRAT et al. (1997) estimaram valores entre 0,27 e 0,40 e MELO et al. (2005) estimaram valores entre 0,22 a 0,36 . Esses baixos valores de $h^{2}$ encontrados no presente estudo, possivelmente, deve-se ao fato de que a maioria dos animais é proveniente de rebanhos de alta produtividade, do Estado São Paulo, onde ocorreu uma intensa seleção para produção de leite no decorrer dos anos.

$A h^{2}$ para P305 foi de 0,26, próxima aos valores relatados na literatura para a raça Holandesa no Brasil (COSTA et al., 2000; FREITAS et al., 2001; FERREIRA et al., 2003; MELO et al., 2005). As $h^{2}$ para as PLDC foram inferiores à obtida para a P305, contrário aos resultados obtidos na literatura, que, em geral, têm relatado maiores estimativas para as PLDC (SWALVE, 1998; MELO et al., 2005).

As estimativas das variâncias genéticas, fenotípicas e residuais das PLDC e da P305 obtidas por meio das médias das análises bicaracterísticas foram bem próximas das estimadas pelas análises unicaracterísticas. Os valores de $\mathrm{h}^{2}$ para as PLDC estimados pelas análises bicaracterísticas foram mais elevados que as estimativas resultantes das análises unicaracterísticas (Tabela 3). Em vários estudos, tem sido levantada a questão da diferença nas estimativas de $h^{2}$ quando estas são provenientes de análises uni ou multicaracterísticas. Tem sido sempre ressaltado que as análises multi-características proporcionam aumento na precisão e na acurácia das estimativas de parâmetros genéticos, quando comparada à uni-característica (MEYER, 1991). Assim como para as análises unicaracterísticas, a maior estimativa de $\mathrm{h}^{2}$ ocorreu na PLDC3. Em geral, as estimativas de $\mathrm{h}^{2}$ relatadas pela maioria dos autores, para vacas Holandesas, têm sido maiores no período intermediário da lactação (MACHADO et al., 1999; FERREIRA et al., 2003). Para a P305 o resultado obtido pela média das estimativas das análises bicaracterísticas foi de 0,27 , similar ao valor encontrado na análise uni-característica.

As estimativas de correlação genética para as PDLC variaram de 0,30 a 1,00 , sendo que as maiores estimativas ocorreram entre controles adjacentes (Tabela 3). Essas estimativas indicam que a seleção para a produção em qualquer controle promoverá ganhos em todas as demais produções, entretanto, as correlações genéticas entre a PLDC1 com as PLDC dos controles finais deve promover menores ganhos nesse período, caso a PLDC1 fosse escolhida como critério de seleção. Os valores de correlação genética do presente estudo foram menores que os relatados por MELO et al. (2005), cujas estimativas variaram entre 0,64 e 1,00, com 71\% das correlações genéticas sendo maiores que 0,95 . Nesse estudo os autores realizaram análises multicaracterísticas, incluindo, simultaneamente, as dez PLDC.

Pode ser visualizado ainda na tabela 3 que as correlações genéticas entre as PLDC do meio da lactação foram maiores que as correlações entre as

Tabela 3 - Estimativas de herdabilidades para as produções de leite no dia do controle (PLDC) e para produção de leite acumulada até 305 dias (P305) (diagonal), correlações genéticas (acima da diagonal) e fenotípicas (abaixo da diagonal) entre as PLDC e entre estas e a P305, em análises bi-características.

\begin{tabular}{|c|c|c|c|c|c|c|c|c|c|c|c|}
\hline & PLDC1 & PLDC2 & PLDC3 & PLDC4 & PLDC5 & PLDC6 & PLDC7 & PLDC8 & PLDC9 & PLDC10 & P305 \\
\hline PLDC1 & 0,12 & 0,86 & 0,81 & 0,80 & 0,56 & 0,51 & 0,46 & 0,49 & 0,43 & 0,30 & 0,63 \\
\hline PLDC2 & 0,57 & 0,16 & 0,99 & 1,00 & 0,95 & 0,89 & 0,78 & 0,64 & 0,55 & 0,60 & 0,85 \\
\hline PLDC3 & 0,48 & 0,68 & 0,22 & 1,00 & 0,88 & 0,87 & 0,85 & 0,77 & 0,69 & 0,62 & 0,93 \\
\hline PLDC4 & 0,39 & 0,57 & 0,64 & 0,18 & 0,88 & 0,99 & 0,83 & 0,72 & 0,73 & 0,62 & 0,97 \\
\hline PLDC5 & 0,34 & 0,49 & 0,58 & 0,63 & 0,15 & 1,00 & 0,89 & 0,81 & 0,75 & 0,73 & 0,89 \\
\hline PLDC6 & 0,27 & 0,44 & 0,52 & 0,55 & 0,62 & 0,14 & 1,00 & 0,98 & 1,00 & 0,91 & 1,00 \\
\hline PLDC7 & 0,26 & 0,40 & 0,47 & 0,49 & 0,56 & 0,61 & 0,16 & 0,91 & 0,90 & 0,79 & 0,93 \\
\hline PLDC8 & 0,24 & 0,34 & 0,41 & 0,45 & 0,47 & 0,55 & 0,61 & 0,19 & 1,00 & 0,93 & 0,91 \\
\hline PLDC9 & 0,23 & 0,35 & 0,40 & 0,42 & 0,46 & 0,48 & 0,55 & 0,64 & 0,20 & 1,00 & 0,94 \\
\hline PLDC10 & 0,17 & 0,23 & 0,30 & 0,32 & 0,32 & 0,35 & 0,42 & 0,49 & 0,60 & 0,19 & 0,87 \\
\hline P305 & 0,50 & 0,65 & 0,71 & 0,71 & 0,70 & 0,71 & 0,74 & 0,71 & 0,74 & 0,63 & 0,27 \\
\hline
\end{tabular}


PLDC nos demais períodos. As correlações genéticas entre as PLDC e a P305 foram todas positivas e elevadas, variando de 0,63 (entre PLDC1 e P305) a 1,00 (PLDC6 e P305). Estes resultados sugerem que a relação entre as PLDC e a P305 é favorável, ou seja, a seleção direta baseada nas produções parciais traria ganhos correlacionados para $\mathrm{P} 305$. Os valores estimados foram similares aos reportados em outros trabalhos com a raça Holandesa. MACHADO et al. (1999) estimaram valores entre 0,78 e 1,00 e FERREIRA et al. (2003) valores entre 0,76 a 1,00 .

As correlações fenotípicas foram todas positivas (Tabela 3 ) e os valores estimados variaram de 0,17 a 0,68 . As correlações residuais variaram de 0,15 (entre PLDC1 e PLDC10) e 0,61 (entre PLDC2 e PLDC3), semelhantes aos resultados relatados por MELO et al. (2005), que variaram de 0,15 a 0,69. Foi observada uma tendência semelhante à correlação fenotípica, ou seja, ocorreu uma gradual diminuição da correlação residual entre os controles à medida que aumentou a distância entre eles. As $\mathrm{h}^{2}$ estimadas para a PLDC1 e também as correlações genéticas entre esta e as demais características mostram que existe uma maior dificuldade em modelar a produção nesse período, pois, além de os animais em estudo serem de primeiro parto, existem vários efeitos relacionados ao estresse no parto que não são considerados nos modelos.

Na tabela 4 encontram-se as eficiências relativas de seleção (ERS) para as PLDC mediante seleção direta para a P305 e eficiências relativas de seleção para a P305, mediante seleção direta para as PLDC, usando-se diferentes números de filhas por touro. Nessa tabela, valores superiores a $100 \%$ indicam maior eficiência de seleção via reposta correlacionada que pela seleção direta.
As ERS para as PLDC, tendo como critério a P305, foram bem maiores que as ERS para as P305, tendo como critério a PLDC, indicando um maior ganho para as produções parciais via resposta correlacionada com a P305. Todavia, quando o número de filhas por touro aumenta, esse ganho diminui porque as diferenças nas acurácias usando as PLDC ou a P305 como critérios de seleção são pequenas. Por exemplo, para a PLDC6, se o touro tem cinco filhas a acurácia é de 0,39 , enquanto que para a P305 é de 0,52. Quando o número de filhas aumenta para 200, a acurácia para a PLDC6 sobe para 0,94, enquanto que para a P305 a acurácia sobe para 0,97 , ou seja, a diferença que era de 0,13 diminuiu para 0,03 .

As PLDC que apresentaram maiores $h^{2}$ (PLDC3, PLDC4 e PLDC9) e as maiores correlações genéticas com a $\mathrm{P} 305$ proporcionaram as maiores eficiências relativas de seleção para a P305, mesmo assim, os valores foram inferiores a $100 \%$. Como as $\mathrm{h}^{2}$ para as PLDC foram menores que para a P305, houve aumento nos ganhos correlacionados para a P305 quando o número de filhas aumentou, usando-se como critério de seleção as PLDC. Observa-se na tabela 4 que como a $\mathrm{h}^{2}$ para a $\mathrm{P} 305(0,27)$ foi maior que para as PLDC, usando-se a P305 como critério de seleção, houve queda na eficiência da resposta para as PLDC, com o aumento do número de filhas por touro. Para a PLDC6, como a correlação genética foi igual à unidade, o aumento do número de filhas por touro proporcionou, quando este número foi maior que 50 filhas, maiores ERS comparadas com as PLDC3, PLDC4 e PLDC9.

Pode-se constatar por meio das ERS que as respostas mais eficientes ocorreriam para as PLDC dos dois controles adjacentes (PLDC2 e PLDC4), diminuindo nos extremos. Como todas as ERS, nesse

Tabela 4 - Eficiências relativas de seleção (ERS) para as produções de leite no dia do controle (PLDC) mediante seleção direta para a produção de leite acumulada até 305 dias (P305) e eficiências relativas de seleção para a P305, mediante seleção direta para as PLDC, usando-se diferentes números de filhas por touro.

\begin{tabular}{|c|c|c|c|c|c|c|c|c|c|c|c|c|}
\hline \multirow{3}{*}{ Característica } & \multicolumn{7}{|c|}{--------ERS para PLDC (\%)-------- } & \multicolumn{5}{|c|}{---------ERS para P305 (\%)--------- } \\
\hline & \multicolumn{7}{|c|}{-------------Número de filhas por touro-------------- } & \multicolumn{5}{|c|}{-------Número de filhas por touro------------- } \\
\hline & 5 & 10 & 20 & 50 & 100 & 200 & 5 & 10 & 20 & 50 & 100 & 200 \\
\hline PLDC 1 & 88,7 & 84,0 & 78,3 & 71,5 & 67,9 & 65,6 & 44,7 & 47,2 & 50,6 & 58,4 & 60,4 & 55,4 \\
\hline PLDC 2 & 105,5 & 101,5 & 96,9 & 91,5 & 88,7 & 87,0 & 68,4 & 71,1 & 74,5 & 78,9 & 81,4 & 83,0 \\
\hline PLDC 3 & 100,9 & 99,3 & 97,5 & 95,4 & 94,3 & 93,7 & 85,7 & 87,0 & 88,7 & 90,6 & 91,7 & 92,3 \\
\hline PLDC 4 & 114,5 & 111,0 & 107,1 & 102,4 & 100,1 & 98,6 & 82,2 & 84,7 & 87,9 & 91,8 & 94,0 & 95,4 \\
\hline PLDC 5 & 113,6 & 108,9 & 103,4 & 96,9 & 93,5 & 91,4 & 69,7 & 72,7 & 76,6 & 81,7 & 84,7 & 86,6 \\
\hline PLDC 6 & 131,5 & 125,6 & 118,6 & 110,2 & 105,8 & 103,1 & 76,0 & 79,6 & 84,3 & 90,7 & 94,5 & 96,9 \\
\hline PLDC 7 & 115,4 & 111,1 & 106,0 & 100,1 & 97,0 & 95,1 & 74,9 & 77,8 & 81,5 & 86,4 & 89,1 & 90,9 \\
\hline PLDC 8 & 105,0 & 102,2 & 99,0 & 95,3 & 93,4 & 92,3 & 78,9 & 81,0 & 83,6 & 86,9 & 88,6 & 89,7 \\
\hline PLDC 9 & 106,1 & 103,7 & 100,9 & 97,7 & 96,1 & 95,1 & 83,2 & 85,2 & 87,5 & 90,4 & 91,9 & 92,9 \\
\hline PLDC 10 & 100,3 & 97,7 & 94,6 & 91,1 & 89,3 & 88,2 & 75,4 & 77,4 & 79,9 & 83,0 & 84,7 & 85,8 \\
\hline
\end{tabular}


caso, foram inferiores a $100 \%$, os ganhos em todas as PLDC, via resposta correlacionada, seriam menores que os obtidos via seleção direta.

Em geral os trabalhos desenvolvidos com os modelos de dimensão finita dos dias do controle tentam propor critérios alternativos de seleção baseados em $\mathrm{h}^{2}$ e correlações genéticas. Por exemplo, no presente estudo, a PLDC3 seria a característica eleita para critério de seleção alternativo à P305, pois sua $\mathrm{h}^{2}$ foi superior às demais PLDC. Os resultados mostraram que, embora haja um ganho para a P305, este será menor que o obtido via seleção direta. Embora não tenha sido explorado no presente estudo, muitos trabalhos têm destacado que o uso de uma PLDC do início ou do meio da lactação como critério poderia antecipar a seleção dos reprodutores, diminuindo o intervalo de gerações e contribuindo para aumentar o ganho genético. Além disso, as filhas de reprodutores, cujas lactações ainda estivessem em andamento, poderiam ser incluídas nas análises, proporcionando aumento na acurácia e, conseqüentemente no ganho correlacionado. Isso de certa maneira já foi demonstrado na tabela 4, ou seja, embora o aumento do número de filhas tenha proporcionado aumento na ERS para a P305, o ganho obtido pela inclusão de maior número de filhas não foi tão evidente.

\section{CONCLUSÕES}

A adoção das produções de leite no dia do controle como critério de seleção poderia antecipar o processo de seleção, encurtando o intervalo de gerações. Entretanto, a seleção para a produção de leite acumulada até 305 dias parece ser o melhor critério de seleção a ser adotado, uma vez que proporciona maiores ganhos genéticos para as produções de leite em, praticamente, todos os controles da lactação.

\section{AGRADECIMENTOS E APRESENTAÇÃO}

À Fundação de Amparo à Pesquisa do Estado de São Paulo (FAPESP) e ao Conselho Nacional de Desenvolvimento Científico e Tecnológico (CNPq), pelo apoio financeiro.

Parte da dissertação apresentada pelo primeiro autor ao Programa de Pós-graduação em Genética e Melhoramento Animal da FCAV - Unesp/Jaboticabal, SP, como parte dos requisitos para a obtenção do grau de Mestre em Genética e Melhoramento Animal.

\section{REFERÊNCIAS}

ARQUIVO ZOOTÉCNICO NACIONAL DE GADO DE LEITE. 20 jun. 2007. Capturado em 23 mar. 2000. On-line. Disponível na Internet: http://www.cnpgl.embrapa.br/azngll
BOLDMAN, K.G. et al. A manual for use of MTDFREML. A set of programs to obtain estimates of variances and covariances. [DRAFT]. Beltsville: US Department of Agriculture, Agricultural Research Service, $1995.125 p$.

COSTA, C.N. et al. Genetic analysis of Holstein cattle populations in Brazil and United States. Journal of Dairy Science, v.83, n.12, p.2963-2974, 2000.

EL FARO,L.; ALBUQUERQUE, L.G. Predição de valores genéticos para a produção de leite no dia do controle e para a produção acumulada até 305 dias. Revista Brasileira de Zootecnia, v.34, n.2, p.496-507, 2005.

FERREIRA, W.J. et al. Avaliação genética de bovinos da raça holandesa usando a produção de leite no dia do controle. Revista Brasileira de Zootecnia, v.32, n.2, p.295-303, 2003.

FREITAS, A.F. et al. Parâmetros genéticos para produção de leite e gordura nas três primeiras lactações de vacas Holandesas. Revista Brasileira de Zootecnia, v.30, n.3, p.709-713, 2001.

FIRAT, M.Z. et al. Univariate analysis of test day milk yields of British Holstein-Friesian Heifers using Gibbs Sampling. Acta Agriculturae Scandinavica, Sect A, v.47, p.213-220, 1997.

INTERBULL. 10 set. 2007. Capturado em 10 set. 2007. Online. Disponível na Internet: http:// /www-interbull.slu.se.

MACHADO, S.G. et al. Genetic parameters of test day milk yields of Holstein cows. Genetics and Molecular Biology, v.22, n.3, p.383-386,1999.

MELO, C.M.R. et al. Parâmetros genéticos para as produções de leite no dia do controle e da primeira lactação de vacas da raça Holandesa. Revista Brasileira de Zootecnia, v.34, n.3, p.796-806, 2005.

MEYER, K. et al. Estimates of genetic parameters for first lactation test day production of Australian Black and White cows. Livestock Production Science v.21, p.177-199, 1989.

MEYER, K. Estimating variances and covariances for multivariate animal models by restricted maximum likelihood. Genetic Selection Evolution, v.23, p.67-83, 1991.

MEYER, K. Estimating covariance functions for longitudinal data using a random regression model. Genetic Selection Evolution, v.30, p.221-240, 1998.

PANDER, B.L. et al. Genetic parameters of day records of British Holstein-Friesian heifers. Animal Production, v.55, p.11-21, 1992.

PTAK, E.; SCHAEFFER, L.R. Use of test day yields for genetic evaluation of dairy sires and cows. Livestock Production Science, v.34, p.23-34, 1993.

SCHAEFFER, L.R.; JAMROZIK, J. Multiple-trait prediction of lactation yields for dairy cows. Journal of Dairy Science, v.79, n.11, p.244-255, 1996.

SWALVE, H.H. The effect of test day models on the estimation of genetic parameters and breeding values for dairy yield traits. Journal of Dairy Science, v.78, n.4, p.929-938, 1995.

SWALVE, H.H. Use of test day records for genetic evaluation. In: WORLD CONGRESS OF GENETICS APPLIED TO LIVESTOCK PRODUCTION, 6., 1998, Armidale, Australia. Proceedings... Armidale: University of New England, 1998. p.295-302.

VAN VLECK, L.D. Selection index and introduction to mixed model methods. Boca Raton: Florida, 1993. 481p. 